\title{
A New-Type Acupuncture Model Based on STM32
}

\author{
Hoiyan Cheung, Litai Lin, Jiawen Chen, Jianwei Zhen, Kaman Yung, Qingyu Ma, Jiaxu Chen* \\ School of Traditional Chinese Medicine, Jinan University, Guangzhou, China \\ Email: 1931479576@qq.com, tai@stu2016.jnu.edu.cn,292983319@qq.com,615336692@qq.com,834959761@qq.com, \\ tmaqingyu@jnu.edu.cn, *chengjiaxu@hotmail.com
}

How to cite this paper: Cheung, H.Y., Lin, L.T., Chen, J.W., Zhen, J.W., Yung, K.M., Ma, Q.Y. and Chen, J.X. (2019) A New-Type Acupuncture Model Based on STM32. Chinese Medicine, 10, 31-38. https://doi.org/10.4236/cm.2019.102004

Received: April 11, 2019

Accepted: June 16, 2019

Published: June 19, 2019

Copyright $\odot 2019$ by author(s) and Scientific Research Publishing Inc. This work is licensed under the Creative Commons Attribution International License (CC BY 4.0).

http://creativecommons.org/licenses/by/4.0/

\begin{abstract}
Background: The foundation of this acupuncture model is to solve the lack of experimental teaching model and student's inadequate ability to identify acupoints in the teaching of acupuncture and moxibustion in traditional Chinese medicine. Methods: In this model we use modern materials to simulate human arms and combination with electronic engineering to create a "Modern Version of Acupuncture Man" and select 2015 undergraduates of Traditional Chinese Medicine School in Jinan University as the research object, to divide those students into two teaching mode which is using acupuncture models or not, to compare their mastery of the acupoint names and position. Results: After the three times different mode teaching and have a test, the average score of using models group was much higher than traditional teaching mode groups $(\mathrm{p}<0.05)$. And all the students expressed that using the model teaching and investigation could better grasp the location and depth of acupoints and enhance their ability of identifying acupoints. Conclusions: The acupuncture models beneficial to the training the acupuncture of Traditional Chinese Medicine students, and also it can make the theory of acupuncture more "Standardization" and "Visualization".
\end{abstract}

\section{Keywords}

Traditional Chinese Medicine, Acupuncture Model, Electronic Devices, STM32

\section{Background}

The foundation of this model is to solve the lack of experimental teaching model and student's inadequate ability to identify acupoints in the acupuncture teaching. The model is designed based on the Wang's bronze human combined with electronic information technology development of the new design and create 1:1 
manikin human arm acupuncture model for TCM teaching demonstration and experimental use for beginners.

The basic core technology of the model is to use REP-601 pressure sensor, and with three layers of silicone rubber material to simulate human muscle toughness to reproduce a new simulation arm model. When receiving the experiment, the acupoint position of the simulated arm is pressed to stimulate the press receptor, which generates electrical signals to the pressure sensor by stimulating the press receptor on the surface. Because the pressure sensor corresponds to the acupoint one by one, the sensor that confirms the change of electric signal can confirm whether the acupoint stimulated at this time is correct. In order to improve the accuracy of the instrument, save cost and diversify the output of results, parallel-to-serial chips are used to improve the utilization of pins of core chips, and STM32 series chips are used as core chips to identify signals and improve the measurement accuracy by combining location algorithm. Because STM32 series chips have the characteristics of high performance, low cost and low power consumption, STM32 series chips are designed through STM32. The image display system displays the relevant information on the acupoint. At the same time, by optimizing the image display system, the teaching of acupuncture and moxibustion in traditional Chinese medicine can be extended to the international market.

\section{Methods}

\subsection{Construction of Embedded Pressure Positioning Data Acquisition Hardware System}

Reconstruction of the human arm meridian model, with reference to the human body model was set up, taking human as the template for the design and construction, the space position of point, to ensure the reliability and practicality of the model, so as to be able to exercise for beginners to identify point ability, and effectively improve the level of water for acupuncture and moxibustion [1]. On the basis of embedded data acquisition hardware system, selection of the core chip and pressure sensor, comprehensive performance, cost and difficulty of developing STM32-F407VET6 is selected as the core control chip [2]. The system includes the main control module, data acquisition module and STM32 display module. The data acquisition module can detect the press on the pressure sensor and display the relevant information of the pressed pressure sensor on the display module when the pressure reaches a certain threshold. Data acquisition module includes pressure testing and data integration to improve the expansibility of the system [3].

\subsection{Redesign of Human Arm Acupoint Model with Three-Layer Material}

Using three layers of material to redesign and change the present situation of single material of the anthropomorphous phantom and inconsistent with human 
body characteristics. VMQ (methyl vinyl silicone rubber) [4], PMMA (methyl methacrylate) [5] and PVC (polyvinyl chloride) [6] silicone rubber materials will be used to re-shape the new-type acupoint model.

Firstly, VMQ is used as the outermost simulated skin structure to cover up the press and other places. In addition, VMQ has excellent high and low temperature resistance, ozone aging resistance and weather resistance. It can keep crack-free under normal temperature stretching condition, and has excellent electrical insulation, odorless and non-toxic. It can produce large deformation under small external force, and can quickly restore its original state after removing external force, those characteristics are suitable for simulating the skin layer.

Thereafter, PMMA as a simulation structure of middle muscles, is suitable to be used as the middle of the model and embedded in pressure sensors because of its low cost, high strength, good toughness, strong rigidity, cold and heat resistance, and good environmental stress cracking and tear resistance.

Finally, using the PVC material into a hollow tubular structure for low level support and simulation of bones, because PVC is low in cost and can produce degeneration, and the bones of the human body is equal to the hardness of the stabilizer in the powerful, is suitable for the bottom.

\subsection{Data Processing and Acupoint Recognition Algorithms Based on Software and Hardware}

\subsubsection{Data Acquisition}

The data acquisition module includes a sensor part and a strobe part. In the sensor part, a thin film strain gauge pressure sensor is used to detect the stress at a specific point on the model, and different resistors are generated according to the different stress. The resistance changes that are difficult to detect can be converted into voltage changes by a simple resistance-voltage conversion circuit. So we choose a simple voltage comparator to quantify the voltage change by detecting the voltage change caused by the resistance change and outputting the state information of the sensor. On the other hand, because the pins of STM32 are limited, it is necessary to multiplex the pins by using the gating device, control the working state of the gating device on the clock, then realize the time division multiplexing of the pins, and effectively use the pins to detect the acupoints.

\subsubsection{Acupoint Recognition}

Because of the complexity of human meridians and acupoints, more sensors may be needed to collect the stimulation of acupoints. The pins of STM32 chips are limited. The pin multiplexing of STM32 chips can be realized by hardware to expand the function of pins effectively. By compressing multiple sets of data, the usage of chips can be reduced and the cost can be reduced. By using the shift registers which are more mature in current technology, the pin multiplexing function can be effectively expanded. Port communication is transformed into serial communication to compress the electrical signals of multiple sensors, thus realizing pin multiplexing of the main control chip. At the same time, the data 
pretreated by hardware system is processed and the stimulated acupoints are determined by efficient acupoint recognition algorithm.

\subsubsection{DP (Data Processing)}

In the initial stage of the system, a simple cyclic scanning method is adopted, that is, a main cycle is constructed inside the system, the control signal of the selected circuit is output cyclically, and the state of multiple pins of the system is detected at the same time. According to the port number of the signal and the control signal of the data selector or multi-channel AD converter, the sensor whose stress changes greatly at this time can be confirmed. The corresponding acupoints and related information can be obtained through the direct image inside the system. If $\mathrm{AD}$ converter is used, the quantized voltage value needs to be processed programmatically to confirm the status information of each pressure sensor at this time (Figure 1).

\subsection{Design and Build a Friendly Human-Computer Interaction System}

\subsubsection{Display Module}

The display module uses TFT touch screen. The color screen is driven by ILI9341, which is simple to drive and can be developed with reference to open source program; it uses high-quality LCD panel with 320X240 resolution, high visual angle and ideal display effect; with touch detection IC-XPT 2046, it can directly expand the function of resistance touch control and improve the simplicity and diversity of human-computer interaction; in addition, the module uses 16-bit LCD 8080. Parallel communication interface, compatible with a variety of general-purpose chips, development difficulties and development costs are in the range of prediction.

\subsubsection{HCI (Human-Computer Interactive Interface)}

After initialization, a simple UI interface is displayed for displaying acupoint information and prompting the operation of the system. The UI interface is realized by repeated refresh, and the resistance touch information is read to adjust the interface. Finally, the acupoint information is refreshed according to the feedback information of the data acquisition module. Because there are many uncommon words in acupoint information, special fonts are needed, so the FLASH of the main control chip of the system needs to be expanded and the corresponding initialization of FLASH is needed.

\subsection{Experiment and Model of Hardware System and Algorithm}

To test and verify the accuracy and sensitivity of the hardware system of data acquisition for press positioning, to find the optimal scheme for optimization in practical application, and to test whether the data processing method and acupoint recognition algorithm can effectively filter the interference factors, optimize the overall structure of the algorithm, and test the reliability and stability of 


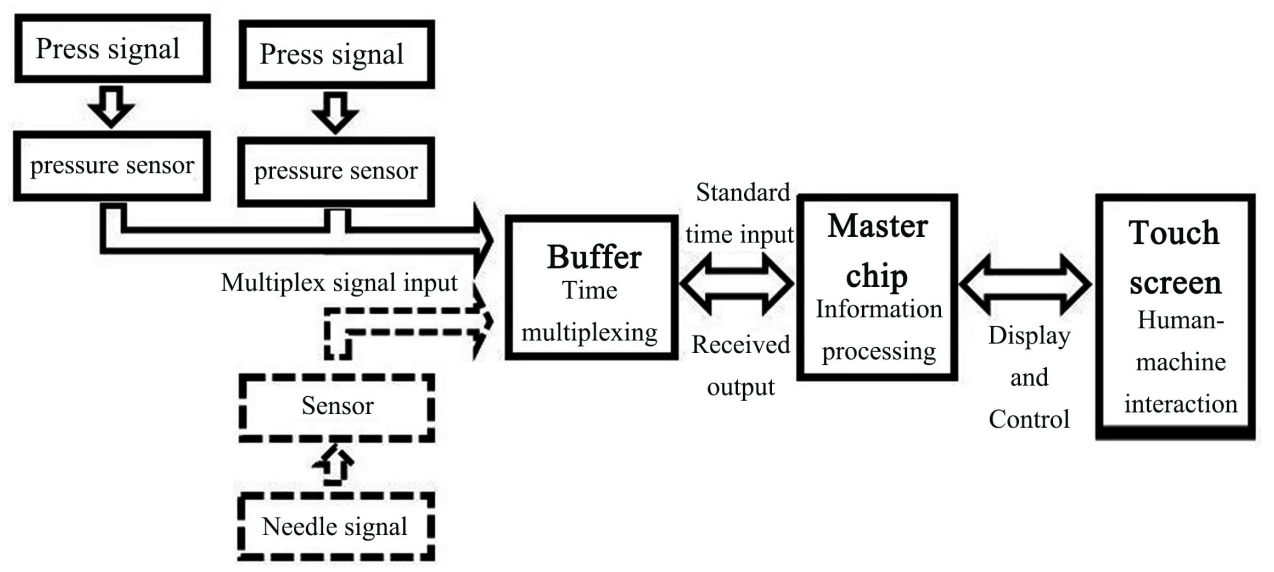

Figure 1. Flow chart of data processing.

the model. It is practicability in teaching, understanding the shortcomings and optimization direction of human-computer interaction system. Find the improvement method of the model, enrich the function of the model and improve the convenience of teaching. Therefore, the system realizes the reading of state information of multiple pressure sensors and the display of acupoint information. That is to say, the state of multiple sensors can be read by the main control chip, and the results can be displayed at the same time.

\section{Results}

With the above method, the team successfully produced a preliminary model of acupoints on the meridians and collaterals of the arm, containing 25 common acupoints on the hands and arms which include their names, meridian tropism and functions. The model was tried out by the students of TCM in our school. We divide the students into two teaching models, which is using acupuncture models or not, to compare their mastery of the acupoint names and position. After the three times, different method of teaching and have a test to assess student grasp corresponding acupoint names and position. The average score of the test of using models group was much higher than traditional teaching mode groups ( $\mathrm{p}<0.05$ ), it shows that using models can make students quick and easier to grasp acupoint. And all the students expressed that using the model teaching and investigation could better grasp the location and depth of acupoints and enhance their ability of identifying acupoints, and more than $90 \%$ students to welcome the teaching model increase to the daily course and examination.

This model was got the third prize of the National Competition for Innovative Design of Traditional Chinese Medicine in Medical Colleges and Universities, and application for the National Utility Model Patent (Application code: 201822068146.6) (Figure 2).

\section{Discussions}

Acupuncture has been one of the most important therapies in traditional Chinese 


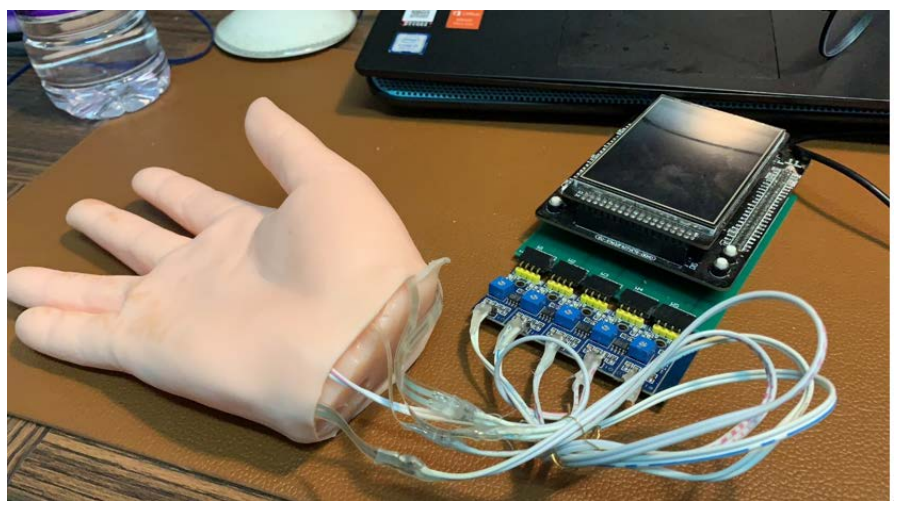

Figure 2. One of the preliminary products.

medicine since ancient times. From the establishment of acupuncture specialty in Tang Dynasty Medical Department to Wang's compilation of "Tongren Acupuncture and MoxibustionTujing" and casting of Tiansheng Bronze Figure in Song Dynasty, and then to 2010, Chinese medicine acupuncturing was listed as "the representative of intangible cultural heritage of mankind", which shows the acupuncture is being valued in the world.

The design of the new acupuncturing teaching model not only includes theory of channels and collateralsmeridian, traditional medicine and anatomy, it also combines modern science and technology such as chemical material technology and electronic circuit. The reconstructed model is more suitable for the true muscular toughness of human body, and the stress is set by the membrane pressure sensor to increase the user's grasp of the position, depth and angle of the acupoints, so that the equipment can better simulate the real feeling of human beings, inspect the student's real ability of acupuncture and moxibustion, and fundamentally standardize the part of acupuncture and acupoints. Therefore, the three-layer material (which include VMQ, PMMA and PVC) can under needling and will not leave holes, it will be great material in the future of the anthropomorphous phantom.

Acupuncture and moxibustion teaching equipment has broad prospects for development. The team has only produced an arm part of the acupuncture model, which has many deficiencies, and has a considerable distance from putting into production. The new needling model introduced in this paper also has some shortcomings in data acquisition, processing and model making technology, and has no function of needling training. In the future, we will be further improved and perfected.

To sum up, science and technology are progressing, and the Chinese medicine industry also needs to improve. We hope that we can build on this acupuncture model and expand it in the future, and join in needle training to develop "Acupuncture and Moxibustion Bronze Man" which truly belongs to the 21st century. Reflecting the perfect combination of science and technology with traditional medicine, catering to the globalization of traditional Chinese medicine, and taking the concept of "inheritance and innovation", to create a new era of tradition- 
al Chinese medicine.

The new type of Acupuncture model based on STM32 is an excellent idea. "Acupuncture and Moxibustion Bronze Man" which are truly belong to the 21st century. STM32 is a family of 32-bit microcontroller integrated circuits by STMicroelectronics. Reconstructed human arm meridian acupoint model is more in line with the characteristics of human hand and buttock muscles, so it can assist students to memorize muscle distribution, thus to deepen the accurate judgment of the depth and angle used in acupoint selection.

The team will use modern materials to simulate human arms and combination with electronic engineering to create a "Modern Version of Acupuncture Man”. The basic core technology of the model is to use REP-601 pressure sensor, and with three layers of silicone rubber material to simulate human muscle toughness to reproduce a new simulation arm model. Because the pressure sensor corresponds to the acupoint one by one, the sensor that confirms the change of electric signal can confirm whether the acupoint stimulated at this time is correct.

Reconstruction of the human arm meridian model, with reference to the human body model was set up, taking human as the template for the design and construction, the space position of point, to ensure the reliability and practicality of the model, so as to be able to exercise for beginners to identify point ability, and effectively improve the level of the acupuncture and moxibustion.

\section{Availability of Data and Material}

The datasets generated and/or analysed during the current study are available in the [NAME] repository [PERSISTENT WEB LINK TO DATASETS].

\section{Funding}

Key Project of National Natural Science Foundation of China (81630104); Guangdong College Students' Innovation and Entrepreneurship Training Program (201810559135).

\section{Foundation Project}

Key Project of National Natural Science Foundation of China (81630104); Education Exchange and Cooperation Project of Ministry of Education of the People's Republic of China (2059999); Guangdong College Students' Innovation and Entrepreneurship Training Program (201810559135).

\section{Authors' Contributions}

Jiaxu Chen design and complete the project, and also revision of articles and give guidance. Hoiyan Cheung conducts the study and writes the paper. Litai Lin and Jiawen Chen analyze and testing of electronic components. Jianwei Zhen and Kaman Yung redesign of acupoint model and collection the acupoint. Qingyu Ma gives technical support. 


\section{Conflicts of Interest}

The authors declare that they have no competing interests.

\section{References}

[1] Cheng, X.N. (2017) Chinese Acupuncture and Moxibustion. Foreign Languages Press, Beijing.

[2] Lindgren, P., Fresk, E., Lindner, M., et al. (2016) Abstract Timers and Their Implementation onto the ARM Cortex-M Family of MCUs. ACM SIGBED Review, 13, 48-53. https://doi.org/10.1145/2907972.2907979

[3] Cady, F.M. (2009) Microcontrollers and Microcomputers Principles of Software and Hardware Engineering. Oxford University Press, USA.

[4] Su, Z.-T. and Wang, J.-H. (2006) Properties of Silicone Rubber at High or Low Temperature. Journal of Aeronautical Materials, 3, 207-212.

[5] Abraham, G.A., Vallo, C.I., Román, J.S. and Cuadrado, T.R. (2004) Mechanical Characterization of Self-Curing Acrylic Cements Formulated with Poly(Methylmethacrylate)/Poly( $\epsilon$-Caprolactone) Beads. Journal of Biomedical Materials Research Part B: Applied Biomaterials, 70, 340-347. https://doi.org/10.1002/jbm.b.30056

[6] Xiong, C.X., Wang, T. and Liu, Q.H. (2004) Study on Preparation and Structure Characterization of Nano-Crystalline Poly (Vinyl Chloride). Journal of Applied Polymer Science, 91, 563-569. https://doi.org/10.1002/app.13152

\section{Abbreviations}

A Family of 32-Bit Microcontroller Integrated Circuits by STMicroelectronics (STM32);

Traditional Chinese Medicine (TCM);

Secure Digital (SD card);

Liquid-Crystal Display (LCD);

Thin-Film Transistor (TFT);

Methyl Vinyl Silicone Rubber (VMQ);

Methyl Methacrylate (PMMA);

Polyvinyl Chloride (PVC);

Unit Interval (UI). 